University of Nebraska - Lincoln

DigitalCommons@University of Nebraska - Lincoln

Martin Centurion Publications

Research Papers in Physics and Astronomy

$9-2006$

\title{
Holographic capture of femtosecond pulse propagation
}

\author{
Martin Centurion \\ University of Nebraska - Lincoln, martin.centurion@unl.edu \\ Ye Pu \\ California Institute of Technology, yepu@sunoptics.caltech.edu \\ Demetri Psaltis \\ California Institute of Technology
}

Follow this and additional works at: https://digitalcommons.unl.edu/physicscenturion

Part of the Physics Commons

Centurion, Martin; Pu, Ye; and Psaltis, Demetri, "Holographic capture of femtosecond pulse propagation" (2006). Martin Centurion Publications. 4.

https://digitalcommons.unl.edu/physicscenturion/4

This Article is brought to you for free and open access by the Research Papers in Physics and Astronomy at DigitalCommons@University of Nebraska - Lincoln. It has been accepted for inclusion in Martin Centurion Publications by an authorized administrator of DigitalCommons@University of Nebraska - Lincoln. 


\title{
Holographic capture of femtosecond pulse propagation
}

\author{
Martin Centurion ${ }^{\text {a) }}$ \\ Center for the Physics of Information, California Institute of Technology, MS 136-93, Pasadena, \\ California 91125 and Department of Electrical Engineering, California Institute of Technology, MS 136-93, \\ Pasadena, California 91125
}

Ye Pu and Demetri Psaltis

Department of Electrical Engineering, California Institute of Technology, MS 136-93, Pasadena, California 91125

(Received 6 March 2006; accepted 8 July 2006; published online 22 September 2006)

\begin{abstract}
We have implemented a holographic system to study the propagation of femtosecond laser pulses with high temporal (150 fs) and spatial resolutions $(4 \mu \mathrm{m})$. The phase information in the holograms allows us to reconstruct both positive and negative index changes due to the Kerr nonlinearity (positive) and plasma formation (negative), and to reconstruct three-dimensional structure. Dramatic differences were observed in the interaction of focused femtosecond pulses with air, water, and carbon disulfide. The air becomes ionized in the focal region, while in water long plasma filaments appear before the light reaches a tight focus. In contrast, in carbon disulfide the optical beam breaks up into multiple filaments but no plasma is measured. We explain these different propagation regimes in terms of the different nonlinear material properties. (C) 2006 American Institute of Physics. [DOI: 10.1063/1.2345469]
\end{abstract}

\section{INTRODUCTION}

The propagation of femtosecond pulses through different media is a subject of great interest. ${ }^{1-5}$ In order to better understand the light-matter interactions, it is important to be able to measure the pulses inside the medium. There are several existing methods to capture ultrafast events; for example, streak cameras, which can operate at picosecond or even subpicosecond resolution, but provide only onedimensional information. Holographic light in flight recording can capture the propagation of short pulses through optical elements; ${ }^{6,7}$ however, this technique captures only the scattering of the beam from a rough surface and cannot capture the beam profile inside the medium. Ultrafast changes in crystals have been measured by recording holograms in the crystals with femtosecond pulses and monitoring the diffraction efficiency. ${ }^{8}$ Pulsed holograms have been used to study laser-induced breakdown with nanosecond time resolution. Light propagation through liquids has been observed by dissolving a fluorescent dye in the material and capturing a side view of the fluorescence. ${ }^{10}$ This method, however, does not provide a time-resolved image. Femtosecond time-resolved optical polarigraphy can be used to observe nonlinear index changes induced through the Kerr effect. ${ }^{11,12}$ This technique captures the transient birefringence induced in the medium, but does not capture isotropic index changes. We have previously demonstrated a holographic technique ${ }^{13}$ that captures laser-induced plasma formation with $150 \mathrm{fs}$ time resolution and recovers amplitude and phase information with a spatial resolution of $4 \mu \mathrm{m}$. Laser pulses with duration of $150 \mathrm{fs}$ probe the changes in the material properties induced by a

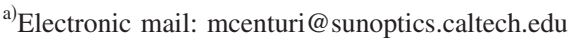

high energy pump pulse. In-line holograms ${ }^{14}$ were captured on a charge-coupled device (CCD) camera and reconstructed numerically.

In this paper we compare the propagation of femtosecond pulses in air, water, and carbon disulfide $\left(\mathrm{CS}_{2}\right)$. We observed dramatic differences in the pulse propagation characteristics depending on the nonlinear properties of the materials. The accuracy of the holographic reconstructions was verified both numerically and experimentally. We have also demonstrated the retrieval of three-dimensional (3D) information by reconstructing the light field at different axial positions, and the capability to record multiple holograms with a single laser shot. The phase recovered from the holograms helps us identify the nonlinear index changes in the material. We have measured both positive and negative index changes. Positive index changes are attributed to the Kerr nonlinearity, while negative index changes are caused by plasma generation.

The Kerr effect generates a change in the index of refraction of the medium which is proportional to the intensity of the pulse. This index change acts as a self-induced lens and causes the beam to focus. A laser pulse will experience self-focusing if its power is higher than a critical value, defined as. ${ }^{15}$

$$
P_{\mathrm{cr}}=\frac{\pi(0.61)^{2} \lambda^{2}}{8 n_{0} n_{2}}
$$

where $\lambda$ is the laser wavelength, $n_{0}$ is the refractive index of the material, and $n_{2}$ is the Kerr coefficient of the material. At the critical power the nonlinearity exactly cancels the effect of diffraction; the beam becomes self-trapped and propagates with a constant diameter. However, this equilibrium between self-focusing and diffraction is unstable, and an additional nonlinearity is necessary for stable propagation of a self- 


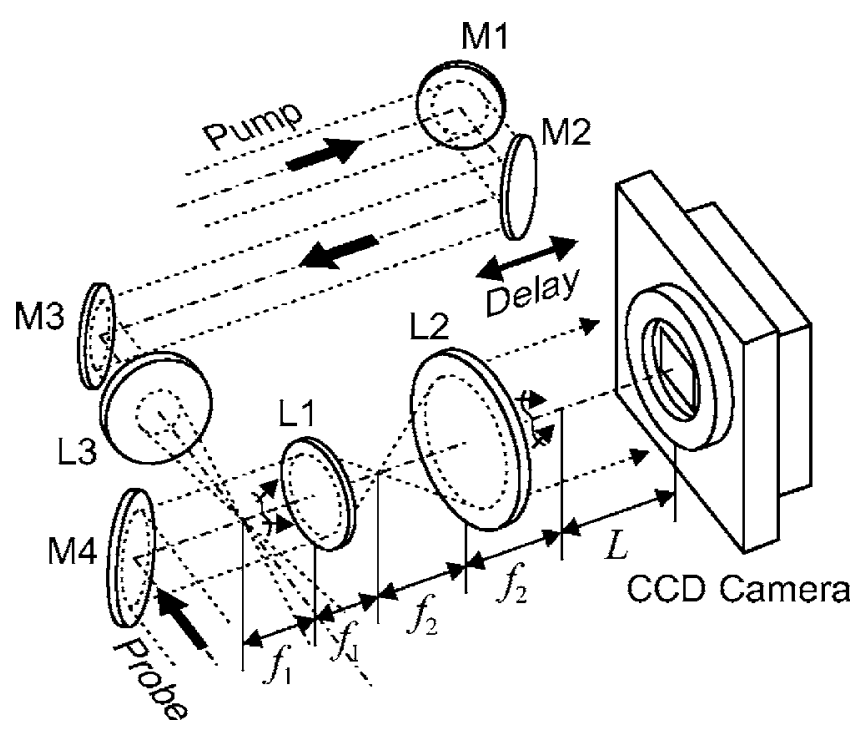

FIG. 1. Experimental setup for single hologram recording.

trapped beam. When the power of the pulses is much greater than the critical power $\left(P>100 P_{\mathrm{cr}}\right)$ the beam will break up into multiple filaments, each one carrying approximately the critical power. ${ }^{15}$ The strength and time constant of the Kerr effect can be inferred from measurements of the index change. If the intensity of the filaments reaches the breakdown threshold, the medium becomes ionized. The density of free electrons can be calculated from the plasma index changes.

\section{EXPERIMENTAL SETUP}

Figure 1 shows the setup used to record an in-line hologram with a femtosecond pulse. A single pulse from a Ti:sapphire laser amplifier operating at $800 \mathrm{~nm}$ wavelength is used to generate the ultrafast event and also to record it. The laser pulses have a duration of $150 \mathrm{fs}$ and a maximum energy of $2 \mathrm{~mJ}$. The beam is approximately Gaussian with a diameter of $5 \mathrm{~mm}$ [full width at half maximum (FWHM)]. The pulse is split into two, with a major portion of the energy going into the pump beam. A delay line (mirrors M1 and M2) is used to synchronize the arrival of the pump and probe pulses. The pump beam is focused with an achromatic lens (L3). A lens with a focal length of $100 \mathrm{~mm}$ is used for the experiments with liquids and a $50 \mathrm{~mm}$ focal length lens is used for the experiments in air. For the experiments with air, ambient air is used and the propagation is observed near the focal region. For the experiments with liquids, a glass cell $(4 \mathrm{~cm}$

a

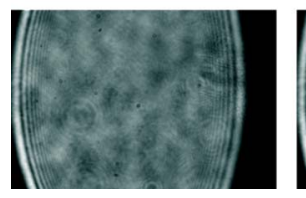

FIG. 2. Background subtraction to remove artifacts due to spatial noise on the optical beam. (a) Image of the reference beam without the signal (the pump beam is blocked). (b) Hologram with the pump beam on. (c) The image in (a) is subtracted from the image in (b) and the mean intensity level is restored to produce a cleaner hologram. in length) is filled with the liquid. The focal point of the lens lies approximately $10 \mathrm{~mm}$ inside the cell. The probe propagates in a direction perpendicular to the pump and captures the interaction of the pump with the material. The image is magnified by a factor of $M=f_{2} / f_{1}$ using lenses L1 and L2, with focal distances of $f_{1}=16.5 \mathrm{~mm}$ and $f_{2}=200 \mathrm{~mm}$, respectively, in a 4-F configuration. A CCD camera (Apogee AP32ME, $2184 \times 1472$ pixels, $6.8 \mu \mathrm{m}$ pixel size) is placed at a distance $L=25-35 \mathrm{~cm}$ from the image plane to capture an in-line hologram. Magnification is used so that the resolution is not limited by the pixel size. The digitized hologram is then numerically reconstructed to retrieve the phase and amplitude changes induced in the probe as it traverses the material. The time resolution of the holograms is limited by the duration of the pulses (150 fs) and the spatial resolution is limited by the numerical aperture of the hologram.

\section{DIGITAL RECORDING AND RECONSTRUCTION OF ON-AXIS HOLOGRAMS}

\section{A. Digital recording and background subtraction}

In holographic recording the optical beams are generally spatially filtered by focusing the beam through a small aperture or pinhole to produce clean uniform beams. However, it is difficult to perform spatial filtering on amplified femtosecond pulses due to strong nonlinearities in the focal region. We have to a large extent removed the effects of spatial noise on the laser beam by first recording an image of the reference light only (the pump beam is blocked) [Fig. 2(a)] and using it as a background that can be subtracted to produce a clean hologram. Figures 2(b) and 2(c) show a hologram before and after background subtraction. The diffraction of the probe is caused by a region of ionized air. The image in Fig. 2(a) is subtracted from the image in Fig. 2(b) and the mean level of intensity is restored to generate the hologram in Fig. 2(c). The resulting hologram contains only the modulation due to the presence of the pump beam.

\section{B. On-axis holograms and the twin image problem}

The use of in-line (Gabor) holograms allows us to record holograms without having to use separate signal and reference pulses with very short coherence lengths. The object is illuminated by a plane wave and the light scattered by the object (signal) interferes with the transmitted light (reference). The intensity of the light field at the recording plane is captured with a CCD camera. The hologram is reconstructed numerically on the computer. Accurate holographic reconstructions can be obtained provided the light scattered by the object is weak compared to the transmitted light, so that the reference beam remains essentially intact. In our experimental setup (Fig. 1) a 4-F system is used to magnify the image so that the reference wave remains a plane wave after going through the 4-F system.

The object is illuminated by a plane wave, which we normalize to be of unit amplitude. The complex light field at the object plane is 


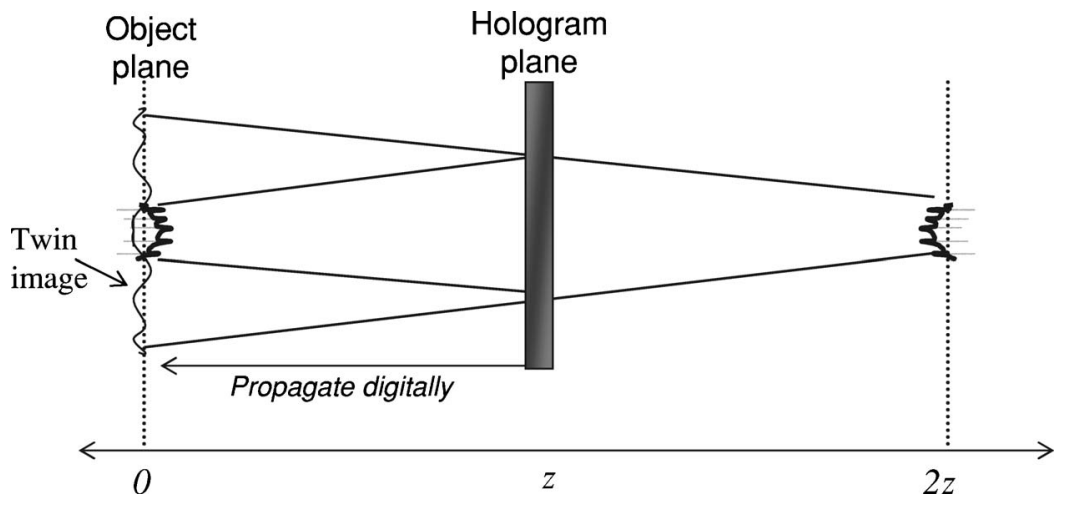

$F_{O}(x, y)=1+S(x, y)$,

where $S(x, y)$ is the complex disturbance of the light field induced by the object, and the phase of the reference is normalized to zero at the object plane. We assume that the area covered by the object is small compared to the area of the illuminating beam (or the area of the camera, whichever is less). This ensures that at the detector the transmitted light field will be much stronger than the object field.

The field at the hologram plane, a distance $z$ from the object plane, is obtained by convolving the object field with the Fresnel convolution kernel, ${ }^{16}$

$$
F_{H}(x, y)=[1+S(x, y)] \otimes h_{z}(x, y),
$$

where $h_{z}(x, y)$ is the Fresnel convolution kernel,

$$
h_{z}(x, y)=\frac{\exp (j k z)}{j \lambda z} \exp \left[\frac{j k}{2 z}\left(x^{2}+y^{2}\right)\right] \text {. }
$$

The convolution can be calculated numerically using fast Fourier transforms. If $F_{H}(x, y)$ is known, the object field can be reconstructed exactly applying the Fresnel convolution kernel in the opposite direction $(-z)$. However, a CCD camera captures the light intensity distribution at the hologram plane,

$$
H(x, y)=\left|F_{H}(x, y)\right|^{2}=\left|[1+S(x, y)] \otimes h_{z}(x, y)\right|^{2} .
$$

If we now try to reconstruct the object field from the intensity measurement using the Fresnel convolution kernel, the reconstructed field becomes

$$
\begin{aligned}
F_{R}(x, y) & =H(x, y) \otimes h_{-z}(x, y) \\
& =1+S(x, y)+S^{*}(x, y) \otimes h_{-2 z}(x, y) .
\end{aligned}
$$

The first term on the right hand side represents the transmitted light (plane wave). The second term is the object field and the third term is a twin image, which is the conjugate of the object field diffracted by a distance of $-2 z$. A term of order square of the object field was neglected. The holographic reconstruction contains the desired amplitude and phase information, but is distorted by the presence of the twin image (Fig. 3). ${ }^{14,17}$ For small objects, the distortion will in most cases appear in the form of fringes around the object. The problem is less severe for small objects and large recording distance $z$, in which case the twin image will appear as background noise around the true object. In the following section we show that for the parameters of the objects we
FIG. 3. Reconstruction of on-axis holograms, both a real and a virtual (twin) image appear in the reconstruction.

\section{Numerical simulation of object reconstruction}

For small objects the presence of the twin image does not significantly affect the accuracy of the reconstruction. In the experiments, the beam breaks up into small filaments with a diameter of $4-15 \mu \mathrm{m}$ and lengths of approximately $1 \mathrm{~mm}$. The overall size of the focal spot that gives rise to the object beam is approximately $50 \mu \mathrm{m}$, consisting of several smaller filaments. The filaments cover only a small fraction of the reference beam that illuminates the CCD camera.

We have performed numerical simulations to predict the distortion induced by the presence of the twin image. We have simulated both a single filament and two filaments in close proximity. The diffraction pattern due to a single filament with a diameter of $8 \mu \mathrm{m}$ and a length of $500 \mu \mathrm{m}$ was calculated for a recording distance of $25 \mathrm{~cm}$. The accumulated phase change for a beam that traverses the filament is $1 \mathrm{rad}$, caused by an index change inside the filament. The magnification, pixel size, and number of pixels are the same as in the experimental apparatus $(M=12,2184$ $\times 1472$ pixels, $6.8 \mu$ mpixel size). The phase change and filament size are comparable to those observed experimentally for plasma filaments generated in air, as described in the following sections (with the difference that the plasma generates a negative index change). The light propagation from the object plane to the recording plane is calculated numerically using the Fresnel convolution Kernel [Eqs. (2) and (3)]. The intensity pattern at the recording plane is used to calculate the reconstruction at the object plane. Figure 4(a) shows a cross section of the phase of the input and reconstructed light field. The reconstruction agrees very well with the simulated filament at the position of the filament. The reconstructed phase is within $5 \%$ of the original in both the peak value and the width. The inset shows a close-up of the filament region. Outside the filament area the reconstruction shows the fringes characteristic of the twin image. We have also simulated two filaments with $8 \mu \mathrm{m}$ diameter separated by $16 \mu \mathrm{m}$ [Fig. 4(b)]. In this case there was also good agreement between the input and the reconstruction, with the characteristic fringes outside of the object area. 
(a)

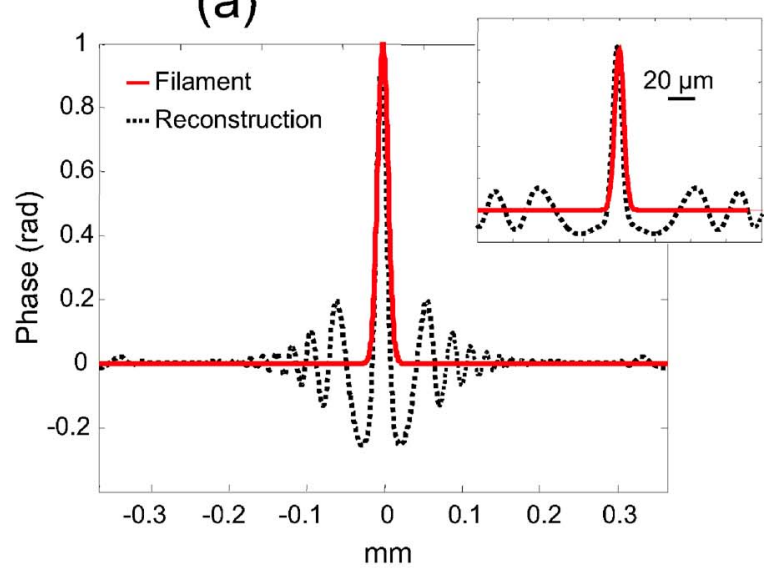

(b)

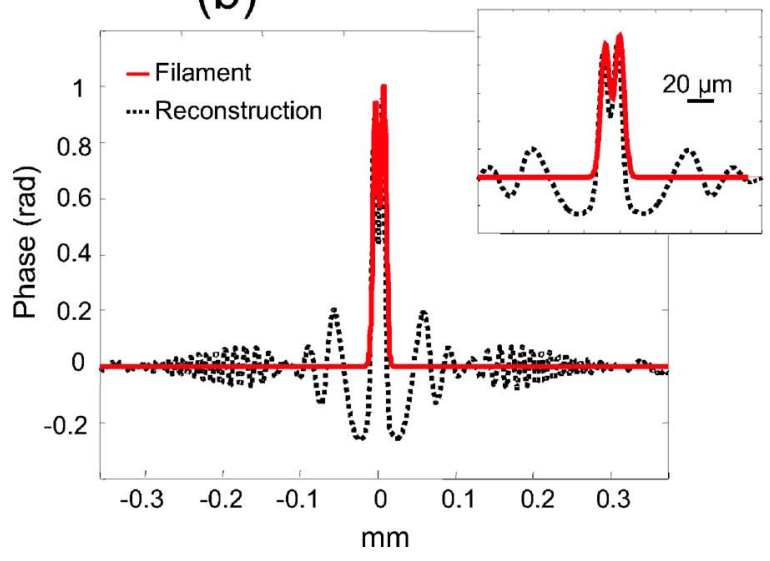

FIG. 4. (Color online) Simulation of phase reconstruction from on-axis holograms. (a) Cross section of simulated (red, solid line) and reconstructed (black, dotted line) phase filament with $8 \mu \mathrm{m}$ diameter. (b) Simulation and reconstruction of double filament. The insets shows a close-up of the filament and reconstruction. In both cases the reconstruction is accurate at the position of the filament.

\section{Comparison of the holographic system with an interferometric phase measurement}

The accuracy of the holographic technique was verified by comparing the phase reconstruction with an interferometric measurement of the phase changes (Fig. 5). A hologram of an air discharge was recorded using the setup in Fig. 1,

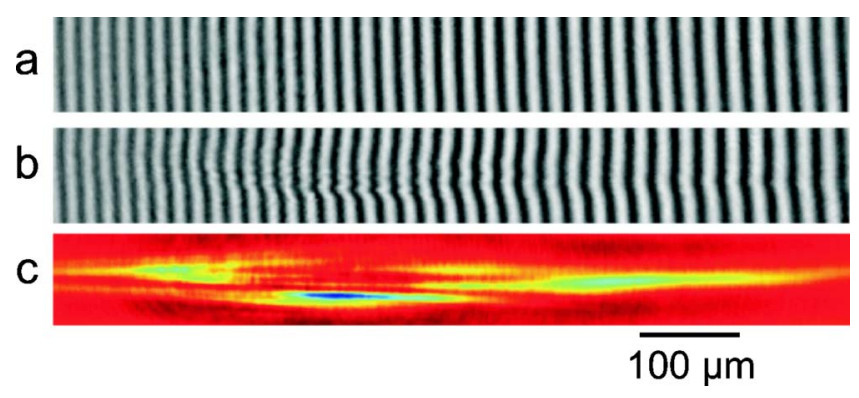

FIG. 5. (Color online) Comparison of on-axis reconstruction with interferometric phase measurement. (a) Interferogram without phase change (the pump is blocked). (b) Interferometric measurement of the the phase change due to the plasma filaments. (c) Holographic reconstruction of the phase change. Red indicates high values and blue low values of the phase. The blue region is the region with the maximum absolute value of phase change. The images are $0.09 \mathrm{~mm}$ (vertical) $\times 0.80 \mathrm{~mm}$ (horizontal). with a recording distance of $L=25 \mathrm{~cm}$. Several plasma filaments are generated in the focal region, which induce a negative phase change in the probe pulse. Multiple experiments with the same conditions resulted in similar patterns of plasma filaments. For the interferometric measurement, the CCD camera is moved to the image plane $(L=0 \mathrm{~cm}$ in Fig. 1 ) and the probe pulse is made to interfere with a uniform reference pulse. The reference pulse is brought from the side at a small angle using a beam splitter and is synchronized to the arrival of the probe pulse. This recording corresponds essentially to an off-axis, image plane hologram with femtosecond pulses. The phase distortions of the signal beam can be measured directly from the fringe dislocations.

Figure 5(b) shows the bending of the interference fringes due to the index change of the plasma and Fig. 5(c) shows the phase reconstructed digitally from the in-line hologram. The phase reconstruction from the in-line hologram looks qualitatively very similar to the phase recorded interferometrically. The regions of high phase change in the holographic reconstruction correspond to the regions where the interference fringes bend more sharply on the interferogram. The maximum phase change is approximately $-1.5 \mathrm{rad}$, which correspond to approximately a $\pi / 2$ phase shift in the interference fringes. The phase reconstructed from the interferometric measurement is in good agreement with the holographic reconstruction. Small differences between the two phase reconstructions are attributable to shot to shot fluctuations in the plasma distribution.

\section{E. Reconstruction of index changes and plasma density}

The nonlinear index changes in the material can be recovered from the phase information in the hologram. A probe that traverses a material with an index change will accumulate a phase change that is proportional to the index change in the material,

$$
\Delta \phi(x, y)=\frac{2 \pi}{\lambda} \int_{0}^{L} \Delta n(x, y, z) d z,
$$

where we assume a probe propagating in the $z$ direction, $\Delta n$ is the index change and $L$ is the length of the index change traversed by the probe. $L$ can be calculated from the size of the filaments, assuming they have a circular cross section. The previous formula can be used to reconstruct the index changes, averaged over the $z$ direction, from the phase measurement,

$$
\frac{\Delta \phi(x, y)}{2 \pi}=\frac{L}{\lambda}\langle\Delta n(x, y)\rangle,
$$

where the term in brackets is the index change averaged in the $z$ direction.

The advantage of the holographic recording is that it captures index changes due to both the Kerr nonlinearty (in general positive) and plasma generation (negative index change), along with changes in the transmittance of the material. The optical Kerr effect causes a change in the index of refraction which is proportional to the intensity of the light, 
a

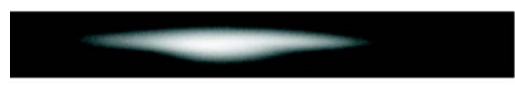

C

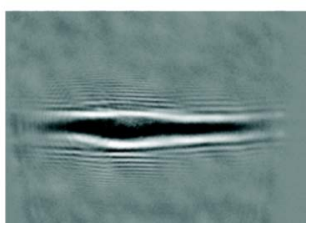

FIG. 6. (Color online) Laser induced discharge in air. (a) Image of laser discharge in air (image size is $0.09 \times 0.80 \mathrm{~mm}^{2}$ ). (b) Hologram of the laserinduced breakdown in air $\left(0.83 \times 1.24 \mathrm{~mm}^{2}\right)$. (c) Reconstruction of the phase changes from the hologram in $(b)\left(0.09 \times 0.80 \mathrm{~mm}^{2}\right)$. Red indicates high values of the phase and blue low values. The pulse propagation is from left to right in the images.

$$
n=n_{0}+n_{2} I,
$$

where $n_{0}$ is the refractive index of the material, $n_{2}$ is the Kerr coefficient of the material, and $I$ is the light intensity. If the intensity of the beam exceeds the breakdown threshold of the material, free electrons are generated. For excitation with ultrashort (femtosecond) pulses, the dominant mechanism for plasma formation is multiphoton absorption. The plasma generated induces a negative index change, which can balance the positive index change of the Kerr effect. The plasma index change is given by

$$
\Delta n=\sqrt{1-\frac{\omega_{p}^{2}}{\omega^{2}}}-1 \approx \frac{-\omega_{p}^{2}}{2 \omega^{2}},
$$

where $\omega=2.36 \times 10^{15} \mathrm{~s}^{-1}$ is the angular frequency of the laser and $\omega_{p}$ is the plasma frequency,

$$
\omega_{p}^{2}=\frac{N e^{2}}{\varepsilon_{0} m},
$$

where $N$ is the electron density, $e=1.6 \times 10^{-19} \mathrm{C}$ is the charge of the electron, $m=9.1 \times 10^{-31} \mathrm{~kg}$ is the mass of the electron, and $\varepsilon_{0}=8.85 \times 10^{-12} \mathrm{C}^{2} \mathrm{~s}^{2} \mathrm{~m}^{-3} \mathrm{k} \mathrm{g}^{-1}$ is the permittivity of free space. The plasma density can thus be calculated if the index change is measured.

\section{EXPERIMENTAL RESULTS}

\section{A. Comparison of pulse propagation in air, water, and $\mathrm{CS}_{2}$}

We first present the results for propagation in air. ${ }^{13} \mathrm{~A}$ pump pulse with energy of $1 \mathrm{~mJ}$ is focused in ambient air. Breakdown of the air is observed around the focal region. Figure 6(a) shows an image of a typical plasma discharge generated in the air. The image shows the light emitted from the discharge, captured with the setup in Fig. 1, by blocking the probe beam and moving the CCD camera to the image plane $(L=0)$. The visible spark is indicative of a high plasma density. Figures 6(b) and 6(c) show the backgroundsubtracted hologram and the numerical phase reconstruction, respectively. The hologram is captured within a picosecond after the pulse traverses the focal region. A strong negative index change is measured in the focal region of the lens, which is attributed to the formation of plasma in the regions of high intensity. The air is ionized through multiphoton absorption; thus the plasma distribution has a nonlinear dependence on the intensity pattern. The amplitude of the probe

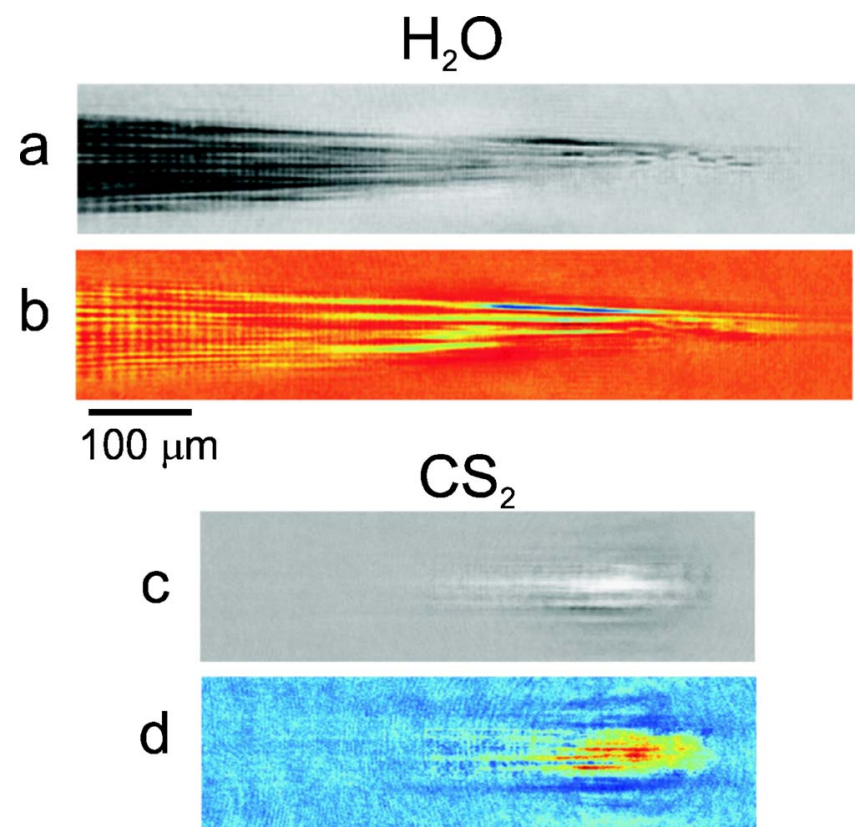

FIG. 7. (Color online) Amplitude and phase reconstructions of femtosecond pulse propagation in liquids. The light pulse propagates from left to right in the images. (a) Amplitude and (b) phase reconstructions for a $700 \mu \mathrm{J}$ focused pulse propagating in water. (c) Amplitude and (d) phase reconstructions for a $50 \mu \mathrm{J}$ focused pulse propagating in $\mathrm{CS}_{2}$. Red corresponds to high values of the phase and blue to low values. The size of the images is 0.15 $\times 0.76 \mathrm{~mm}^{2}$ for (a) and (b) and $0.15 \times 0.54 \mathrm{~mm}^{2}$ for (c) and (d).

light is only weakly modulated by the presence of the plasma. The plasma region has a maximum width of $50 \mu \mathrm{m}$ and length of $800 \mu \mathrm{m}$. The free electrons are concentrated in filaments with diameters of $5-10 \mu \mathrm{m}$. The minimum measured filament size is close to the resolution limit of the system, so it is possible that some filaments are smaller. The measured index change inside the plasma filaments varies from $\Delta n=2 \times 10^{-3}$ to $2 \times 10^{-2}$. The density of electrons in the plasma filaments, calculated using Eqs. (10) and (11), varies between $7 \times 10^{18}$ and $7 \times 10^{19} \mathrm{~cm}^{-3}$. Most of the plasma generated is concentrated in the filaments, which are confined to the focal region. The mean plasma density over the focal volume is on the order of $10^{17} \mathrm{~cm}^{-3}$. The peak plasma densities that we measured are two orders of magnitude higher than those reported for long range filaments, ${ }^{18,19}$ i.e., filaments that propagate in air for several meters. While the plasma in long range filaments results from a balancing between diffraction, Kerr self-focusing and plasma defocusing, in our experiments higher plasma densities are caused by the strong focusing geometry.

We have also recorded holograms of pulses propagating in water and $\mathrm{CS}_{2}$. Figure 7 shows (a) the amplitude and (b) phase reconstruction for a $700 \mu \mathrm{J}$ pulse propagating in water, while (c) and (d) display the amplitude and phase reconstructions for a $50 \mu \mathrm{J}$ pulse propagating in $\mathrm{CS}_{2}$. Different pulse energies were used because the Kerr nonlinearity is stronger in $\mathrm{CS}_{2}$.

For a pulse propagating in water, the beam has a spatial profile which follows the linear focusing of the lens, while inside this envelope the beam breaks up into multiple filaments before reaching the focal plane [Figs. 7(a) and 7(b)]. The propagation direction of the filaments is determined by 
the local wave vector. More specifically, since the beam has a spherical wave front, the filaments propagate inside a cone determined by the focusing lens. No filaments were observed after the focal plane. In Figs. 7(a) and 7(b) the pulse is at the leading edge of the filaments, while the dark trace to the left forms after the pulse has traversed the region. The trace is characterized by a decrease in the transmission of the probe beam of approximately $30 \%$, while the phase change is too weak to be reconstructed accurately. The decrease in transmission was also observed when the plasma region was directly imaged on the CCD camera, in good agreement with the holographic reconstruction. The filaments at the leading edge generate large negative phase changes and small amplitude changes. The size of the filaments is approximately $5 \mu \mathrm{m}$ and the calculated index change is $\Delta n=-10^{-2}$. As in the propagation in air, the negative index change results from the formation of plasma in the regions of highest intensity. This index change corresponds to a plasma density of 3 $\times 10^{19} \mathrm{~cm}^{-3}$ inside the filaments. Vapor bubbles were observed near the focal region in the water (long after the experiment), due to localized boiling of the liquid. Bubbles are expected to appear for plasma densities above $10^{18} \mathrm{~cm}^{-3} .{ }^{20}$ The plasma filaments appear 1-2 $\mathrm{mm}$ before the focal plane, in contrast to the experiment in air where the plasma is confined to the focal region. The dark traces result from plasma filaments scattering or absorbing light from the probe pulses. There is a delay of approximately 2 ps between the initial arrival of the pump pulse and the onset of the absorption/ scattering. Initially there is a clear evidence of a phase change, followed by the increased absorption. From further pump and probe experiments we determined a decay constant of the plasma trace of approximately $0.5 \mathrm{~ns}$, much longer than the time it takes to generate it. In addition, conversion of the pump pulse into a white light continuum ${ }^{21}$ was observed at the output of the glass cell containing the water.

Similar experiments were performed in $\mathrm{CS}_{2}$. We measured positive phase changes and an increase in intensity of the probe beam near the center of the pulse position [Figs. 7(c) and 7(d)]. The phase change corresponds to the positive index change generated through the Kerr effect. The region of nonlinear index change acts as a focusing lens on the probe beam to generate the observed intensity profile. As opposed to propagation in air or water, no plasma was observed in $\mathrm{CS}_{2}$. In the absence of a negative index change the optical filaments will continue to self-focus until they reach a size comparable to the wavelength and collapse; however, in $\mathrm{CS}_{2}$ we have observed that the filaments reach a stable diameter. We believe there is a saturation of the nonlinear index change (possibly through a fifth order nonlinearity) which causes a clamping of the intensity in the filaments. ${ }^{5,22}$ The formation of a very weak plasma (with a density below the sensitivity of our technique) inside the filament could also contribute to the intensity clamping. In the reconstructed phase [Fig. 7(d)] we have observed multiple filaments with diameters of $8-15 \mu \mathrm{m}$ and an index change of approximately $\Delta n=7 \times 10^{-4}$. It is difficult to determine the size of the filaments accurately because they are packed very close together, separated only by $5-10 \mu \mathrm{m}$. At the leading edge of the pulse, the pump and probe pulses temporally and spa-
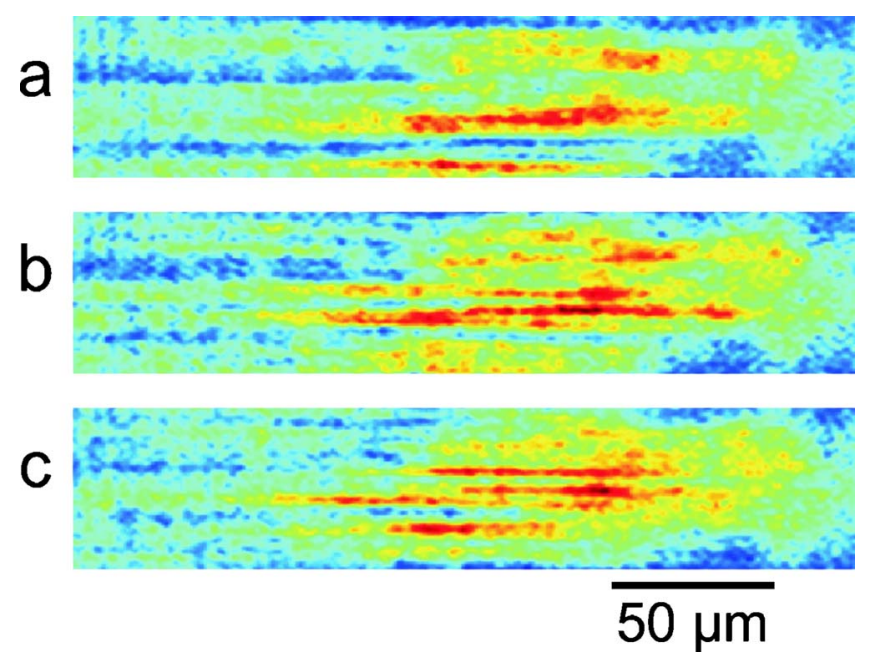

FIG. 8. (Color online) Three depth slices of the object reconstructed from a single hologram. The images show the phase of the light at three different planes by changing the reconstruction distance in steps of $40 \mu \mathrm{m}$. The cross sections reveal the 3D structure of the filamentation pattern. (a) A filament in the lower part of the image comes to focus while the rest of the structure is out of focus. (b) A pattern of filaments appears in the center region and the filament in the lower part of the images is now out of focus. (c) A different set of filaments comes to focus in the central region of the image. The size of the images is $0.05 \times 0.24 \mathrm{~mm}^{2}$.

tially overlap, giving rise to the region with a stronger index change in Fig. 7(d). A trail of weaker index change is also observed behind the pulse. No negative index changes (plasma) were observed in the trail. The stronger signal at the overlap of the two pulses is due to the instantaneous (electronic) Kerr response of the material, while the trail is due to the slower (molecular) response. The length of the trail is consistent with the known lifetime of the index change of approximately 2 ps. $^{3}$

\section{B. Reconstruction of 3D information}

The beam profile in $\mathrm{CS}_{2}$ contains multiple filaments distributed in 3D which cannot be resolved by reconstructing a single two-dimensional (2D) projection of the beam. The hologram of pulse propagation in $\mathrm{CS}_{2}$ was digitally refocused at different planes to reveal the three-dimensional structure of the beam profile. The estimated axial resolution of the reconstruction is $20 \mu \mathrm{m}$ and is limited by the numerical aperture of the hologram. In the case of beam propagation through $\mathrm{CS}_{2}$ the diameter of the filaments is $8-15 \mu \mathrm{m}$, while the diameter of the whole beam is close to $50 \mu \mathrm{m}$. Due to the limited depth resolution the filaments will appear elongated in the axial direction. We have numerically reconstructed the hologram at three different planes, separated by a distance of $40 \mu \mathrm{m}$ between each plane, to obtain three depth slices (cross sections) of the beam profile. Figure 8 shows the phase of the reconstructed light field at the three different planes. Each reconstruction reveals a different filamentation pattern as different filaments come to focus. A single filament near the bottom of the image is in focus in Fig. 8(a), while the rest of the filaments are out of focus. In Figs. 8(b) and 8(c) the filament at the bottom is out of focus and different filaments come to focus in the central region for the two 


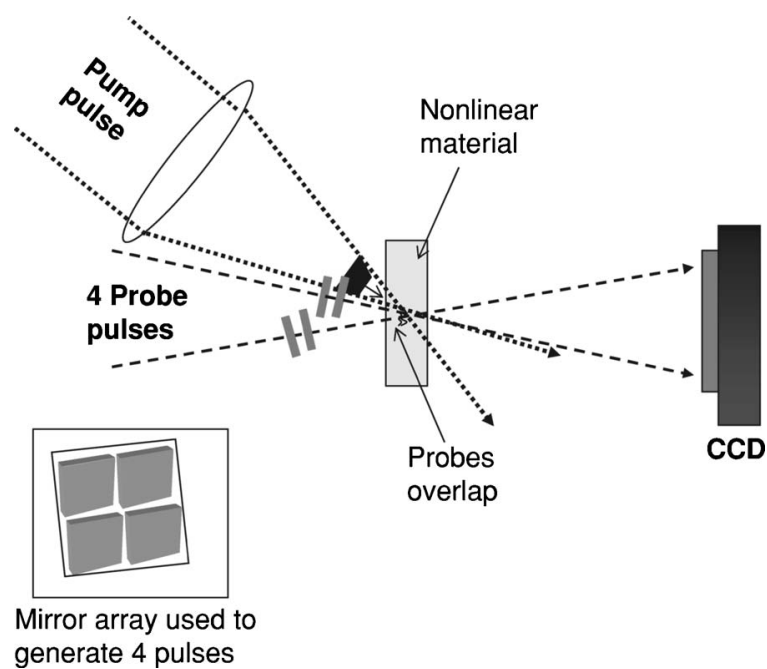

FIG. 9. Experimental setup for holographic movie recording. The angle between the probes pulses is exaggerated for visual clarity.

different refocusing distances. A higher numerical aperture could be used for a full 3D reconstruction of the filamentation pattern.

\section{Multiple frame capture (holographic movie)}

We compare the propagation dynamics in water and $\mathrm{CS}_{2}$ by spatially multiplexing a time sequence of four holograms in a single frame of the CCD camera. The setup in Fig. 1 was modified to record a time sequence of four holograms with a single laser pulse. Mirror M4 is replaced with a mirror array consisting of four mirror segments (Fig. 9), each mirror having independent controls for translation and tilt in two dimensions. Four probe pulses are generated by reflecting a single pulse off the mirror array. The input beam is expanded and only the central region is used such that the intensity of the probe beams is uniform. An aperture is placed at the mirror array to limit the probe beams to a square size with a width of $5 \mathrm{~mm}$. The position of the mirrors controls the relative time delay between the probes, while the angle controls the propagation direction. The four probes are made to spatially overlap in the interaction region (and then spatially separate on the recording plane). Each probe pulse samples the event at a time set by the displacement of the mirror. The relative time delay between the probes can be adjusted to match the time window of interest, while a delay line on the pump arm synchronizes the arrival of pump and probe pulses. The probes propagate in a direction perpendicular to the CCD camera, while the pump traverses the material at an angle $\left(22^{\circ}\right.$ for the experiments in water and $18^{\circ}$ in $\left.\mathrm{CS}_{2}\right)$. The angle difference is in the horizontal direction, as observed on the camera. Smaller angles lead to a stronger signal as the phase change accumulates over a longer distance. If the pump pulse leaves a trail in the material which lasts longer than the time window of the experiment (a few picoseconds), then a probe pulse arriving after the pump will capture the entire trail. An instantaneous effect, however, can only be captured when the pulses overlap temporally and spatially. After traversing the interaction region the probe beams propagate a distance of $200 \mathrm{~mm}$ to the CCD camera (no

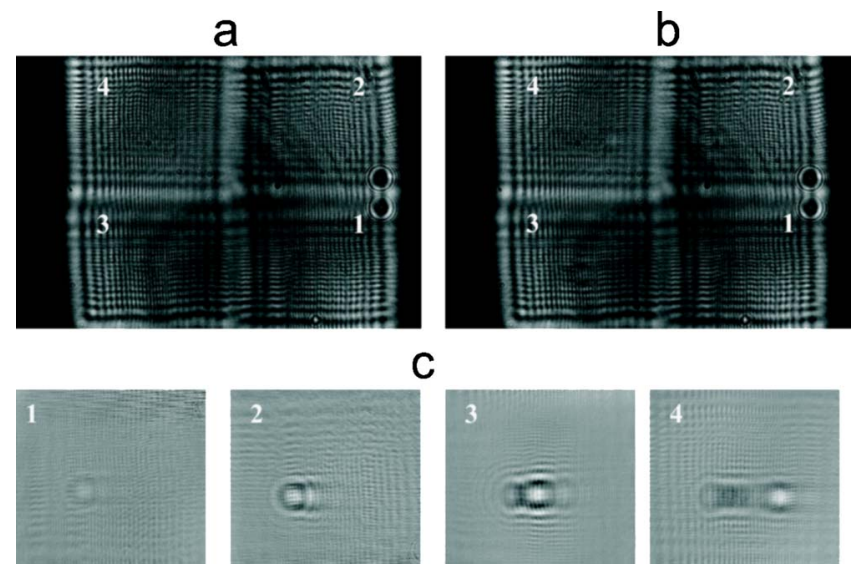

FIG. 10. Time sequence of pulse propagation in water on a single frame of the CCD camera. (a) Four plane-wave probe beams on the CCD camera (the pump beam is blocked). The fringes result from an aperture used to shape the beams. (b) Four on-axis holograms with relative time delays of 0, 0.7, 1.3 , and $2.3 \mathrm{ps}$. A weak modulation can be seen near the center of each frame. (c) The individual holograms (subframes) after the background is subtracted.

lenses are used). We set the separation angle between the probe pulses sufficiently small $\left(1.4^{\circ}\right)$ so that the events are captured at approximately the same angle. In order to spatially separate the four holograms in the CCD sensor, the effective angular aperture of each individual hologram is limited to the separation angle between the probe pulses. The spatial resolution of each hologram $(60 \mu \mathrm{m})$ is determined by the angular aperture.

Figure 10 shows a time sequence of four holograms of pulse propagation in water captured in a single frame of the CCD camera. A 150 fs pump pulse with $300 \mu \mathrm{J}$ energy is focused inside a glass cuvette filled with water. A background image is captured with only the probe beams [Fig. 10(a)] and used to remove artifacts in the hologram caused by modulation of the probe beams. The diffraction fringes in the probes are caused by reflection from the square segments in the mirror array. The four beams are spatially separated on the CCD sensor and temporally separated by relative time delays of $0,0.7,1.3$, and 2.3 ps for subframes $1,2,3$, and 4 , respectively. A second image is captured with the pump beam overlapping with the probes inside the medium [Fig. 10(b)]. The holograms are corrected by subtracting the image in Fig. 10(a) from the image in Fig. 10(b) and restoring the background light intensity to the mean intensity in Fig. 10(b). Figure 10(c) shows the resulting background subtracted holograms, after cutting the four subframes from the single CCD frame. The modulation that is left is due only to the interaction of pump and probe beams and can be used to reconstruct the amplitude and phase of the probe pulses.

The reduced hologram area and the reduced spatial resolution lead to more distortion in the reconstructions. In this case the presence of the twin image can significantly affect the accuracy of the reconstruction. We have used an iterative algorithm to numerically remove the effect of the twin image on the reconstruction and recover amplitude and phase. ${ }^{23}$ The algorithm takes advantage of the fact that the object is small (compared to the hologram area) and is illuminated by a known wave form, in this case a plane wave. 


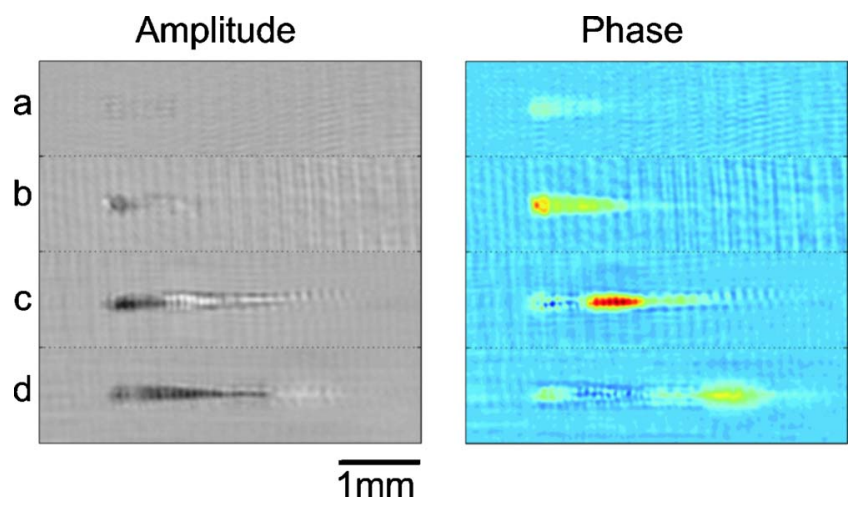

FIG. 11. (Color online) Holographic amplitude (left) and phase (right) reconstructions of femtosecond pulse propagation in water. The relative time delays are (a) 0 , (b) 0.7 , (c) 1.3 , and (d) 2.3 ps. The maximum phase change is $0.6 \mathrm{rad}$ in (c). Each subframe is $4 \mathrm{~mm}$ (horizontal) by $1 \mathrm{~mm}$ (vertical).

Figure 11 shows the amplitude and phase reconstructed from the holograms of pulse propagation in water in Fig. 10(c). For the experiments in $\mathrm{CS}_{2}$ the pulse energy is reduced to $50 \mu \mathrm{J}$; Fig. 12 shows the reconstructed amplitude and phase for probe pulses with relative time delays of $t=0$, 1.0, 2.0, and $3.0 \mathrm{ps}$. In the first frame [Figs. 11(a) and 12(a)] the probe pulse interacts only with the leading edge of the pump and a weak signal is captured. The second and third holograms [Figs. 11(b), 11(c), 12(b), and 12(c)] capture the pulse before the focal point, while the fourth frame [Figs. 11(d) and 12(d)] captures the pulse after the focal point. The Kerr index change in water becomes visible with this setup (as opposed to the single shot setup in Fig. 1) due to the longer interaction length of pump and probe pulses. The nonlinear Kerr index change in water is instantaneous (on the order of a femtosecond), so a snapshot of the propagating pulse is captured. In contrast, the Kerr effect in $\mathrm{CS}_{2}$ has both instantaneous and noninstantaneous contributions, so as the pulse traverses the liquid it leaves a trace of positive index change. The trace shows the pulse entering the medium, focusing to a small spot and defocusing. Using longer time delays between the pump and the probes we measured a decay time constant of the noninstantaneous index change of $1.7 \mathrm{ps}$, in good agreement with the values reported in the literature. $^{3}$

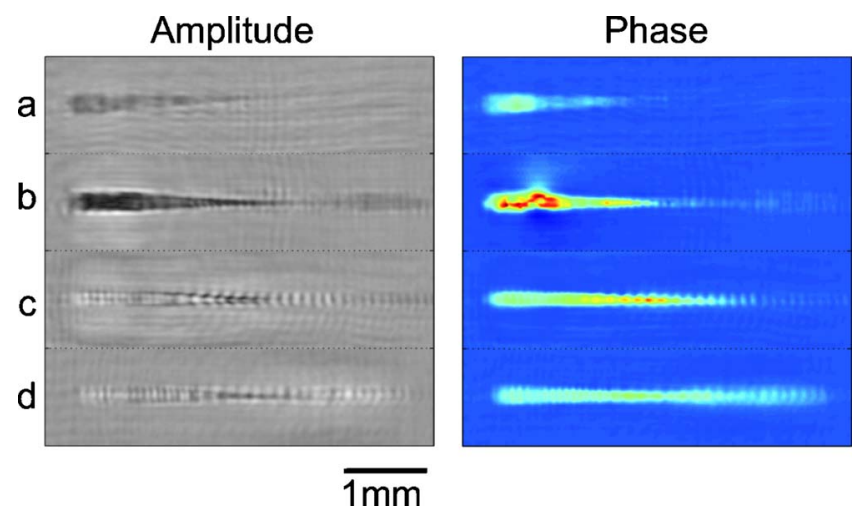

FIG. 12. (Color online) Holographic amplitude (left) and phase (right) reconstructions of femtosecond pulse propagation in $\mathrm{CS}_{2}$. The maximum phase change is $4 \mathrm{rad}$ in (b) and $2 \mathrm{rad}$ in (c). The relative time delays are (a) 0 , (b) 1.0 , (c) 2.0 , and (d) $3.0 \mathrm{ps}$. Each subframe is $4 \mathrm{~mm}$ (horizontal) by $1 \mathrm{~mm}$ (vertical).
The diameter of the beam in the focal region is approximately $150 \mu \mathrm{m}$ in water [Fig. 11(c)] and $60 \mu \mathrm{m}$ in $\mathrm{CS}_{2}$ [Fig. 12(c)]. The Kerr index changes in the focal region are 6 $\times 10^{-4}$ in $\mathrm{CS}_{2}$ and $1 \times 10^{-4}$ in water. Note that even though the pulse energy was six times higher in water, the index change in $\mathrm{CS}_{2}$ is greater. The phase reconstruction in water also shows a trail of negative index change, which corresponds to the long-lasting plasma trail observed with the high resolution holograms. The dark regions in the amplitude reconstructions in Fig. 11 correspond to the plasma trail. The weak amplitude changes in Figs. 12(a), 12(c), and 12(d) result from the long depth of focus of the system and possibly numerical errors. The dark region in the amplitude reconstruction in Fig. 12(b) is due to a strong phase change experienced by the probe. The index change acts like a lens which focuses the light in a short distance so that it defocuses quickly and some of the light misses the detector. The amplitude of the light in the dark region is approximately 55\% of the background light level. We have thus observed both positive (Kerr) and negative (plasma) index changes in water, while propagation in $\mathrm{CS}_{2}$ was characterized by a longlasting positive index change and no plasma generation.

\section{DISCUSSION}

We have presented a holographic method to measure ultrafast changes in material properties with femtosecond time resolution. The amplitude and phase information stored in the holograms allow us to recover changes in the absorption and index of refraction with high temporal and spatial resolutions. We have measured changes in the index of refraction due to the Kerr effect (positive) and plasma formation (negative) and reconstructed their spatiotemporal evolution. We have applied our method to study the propagation of femtosecond pulses in a gas (air) and liquids (water and $\mathrm{CS}_{2}$ ). The technique could also be used to study transient and permanent changes in solids.

We have observed laser-induced ionization in air and water. The spatial distribution of the plasma was reconstructed from the phase information in the holograms. The plasma in the air is confined to a small region near the focal plane, while plasma filaments appear in water long before the focal spot. The different behavior is attributed to the larger Kerr coefficient in water. The Kerr coefficients [Eq. 2.3] are $n_{2 \text { (air) }}=3 \times 10^{-19} \mathrm{~cm}^{2} / \mathrm{W}$ for air $^{24}$ and $n_{2 \text { (water) }}$ $=4 \times 10^{-16} \mathrm{~cm}^{2} / \mathrm{W}$ for water. ${ }^{15}$ The self-focusing nonlinearity causes the power to be concentrated in small filaments which reach an intensity level above the ionization threshold. The ionization thresholds are $I_{\text {th }}=2 \times 10^{13} \mathrm{~W} / \mathrm{cm}^{2}$ in air $^{25}$ and $I_{\mathrm{th}}=1.2 \times 10^{13} \mathrm{~W} / \mathrm{cm}^{2}$ in water. ${ }^{26}$ The plasma in air first forms along the propagation axis and then broadens as the whole beam is focused above the threshold. We have also reconstructed both positive and negative index changes in the same hologram using the experimental setup in Fig. 9 (where the pump and probe have a longer interaction length). The positive index change corresponds to an instantaneous Kerr index change that travels with the pump pulse, and the negative index change is due to the long-lasting plasma trail behind the pulse. We also demonstrated the capability to 
record a time sequence of four holograms with a single laser pulse. Single shot recording becomes necessary for unrepeatable events and can also be used to minimize material damage.

In the case of propagation in $\mathrm{CS}_{2}$ we did not observe laser-induced ionization. A positive index change due to the Kerr effect was measured. The Kerr coefficient in $\mathrm{CS}_{2}$ $\left[n_{2\left(\mathrm{CS}_{2}\right)}=3 \times 10^{-15} \mathrm{~cm}^{2} / \mathrm{W}\right]$ (Ref. 27) is larger than that in water and has a different temporal response. The time resolution in the holograms allowed us to capture both the instantaneous (electronic) and delayed (molecular) Kerr responses of the material. The capability of holography to reconstruct the light field at different planes was used to reveal the 3D structure of the filamentation pattern. The light beam breaks up into multiple filaments long before reaching the focal point, preventing the intensity from reaching the ionization threshold. The number of filaments is much higher in $\mathrm{CS}_{2}$ than in water so that each filament carries less power; in addition, the maximum intensity in the filaments is clamped by the effect of a defocusing nonlinearity.

Using a CCD camera as the recording medium provides a high enough sensitivity to capture a hologram with only a small fraction of the energy of a single laser pulse. This allowed us to use most of the pulse energy to generate the nonlinearities and even to record multiple holograms with a single pulse. The temporal resolution in our system is limited by the duration of the laser pulses, while the spatial resolution is determined by the wavelength and the numerical aperture of the hologram. The main advantage of our system is the capability to capture and differentiate multiple nonlinear effects without an a priori knowledge of the behavior of the medium. The system can measure the strength and time response of the Kerr nonlinearity, along with the spatiotemporal plasma distribution during laser-induced ionization.

\section{SUMMARY}

We have demonstrated a holographic system to capture the propagation of femtosecond laser pulses with high temporal $(150 \mathrm{fs})$ and spatial resolutions $(4 \mu \mathrm{m})$. The holographic reconstruction allows us to recover both positive (Kerr) and negative (plasma) index changes and to calculate the spatially resolved electron density of the plasma. We have studied the propagation of pulses in air, water, and $\mathrm{CS}_{2}$ and seen dramatic differences depending on the material properties.

The advantage of a holographic setup is that it provides amplitude and phase information, and the possibility to reconstruct 3D information. The holograms were recorded on a CCD camera and reconstructed numerically. The accuracy of the reconstructions was verified both numerically and experimentally. Finally, we have also demonstrated the capability to record a time sequence of four holograms in a single shot experiment using spatial multiplexing.

\section{ACKNOWLEDGMENTS}

We would like to thank Zhiwen Liu and Theodor W. Hänsch for helpful discussions. This research was supported by the Defense Advanced Research Projects Agency Center for Optofluidic Integration. Funding for one of us (M.C.) was provided through the Center for the Physics of Information as part of the Caltech Information Science and Technology initiative.

${ }^{1}$ A. Braun, G. Korn, X. Liu, D. Du, J. Squier, and G. Mourou, Opt. Lett. 20, 73 (1995).

${ }^{2}$ R. Fork, C. Shank, C. Hirlimann, R. Yen, and W. J. Tomlinson, Opt. Lett. 8, 1 (1983).

${ }^{3}$ N. Akozbek, A. Becker, and S. L. Chin, Laser Phys. 15, 607 (2005).

${ }^{4}$ A. Dubietis, G. Tamosauskas, I. Diomin, and A. Varanavicius, Opt. Lett. 28, 1269 (2003).

${ }^{5}$ M. Centurion, Y. Pu, M. Tsang, and D. Psaltis, Phys. Rev. A 71, 063811 (2005).

${ }^{6}$ N. Abramson, Appl. Opt. 22, 215 (1983).

${ }^{7}$ M. Yamagiwa, A. Komatsu, Y. Awatsuji, and T. Kubota, Opt. Express 13, 3296 (2005).

${ }^{8}$ H. T. Hsieh, D. Psaltis, O. Beyer, D. Maxein, C. von Korff Schmising, K. Buse, and B. Sturman, Opt. Lett. 30, 2233 (2005).

${ }^{9}$ Z. W. Liu, M. Centurion, G. Panotopoulos, J. Hong, and D. Psaltis, Opt. Lett. 27, 22 (2002).

${ }^{10}$ H. Schroeder and S. L. Chin, Opt. Commun. 234, 399 (2004).

${ }^{11}$ M. Fujimoto, S. Aoshima, M. Hosoda, and Y. Tsuchiya, Opt. Lett. 24, 850 (1999).

${ }^{12}$ M. Centurion, Y. Pu, and D. Psaltis, Opt. Express 13, 6202 (2005).

${ }^{13}$ M. Centurion, Y. Pu, Z. W. Liu, D. Psaltis, and T. W. Hansch, Opt. Lett. 29, 772 (2004).

${ }^{14}$ D. Gabor, Nature (London) 161, 777 (1948).

${ }^{15}$ R. Boyd, Nonlinear Optics (Academic, San Diego, CA, 2003).

${ }^{16}$ J. Goodman, Fourier Optics (McGraw-Hill, New York, 1996).

${ }^{17}$ G. Liu and P. D. Scott, J. Opt. Soc. Am. A 4, 159 (1987).

${ }^{18}$ L. Berge and A. Couairon, Phys. Rev. Lett. 86, 1003 (2001).

${ }^{19}$ S. Tzortzakis, L. Berge, A. Couairon, M. Franco, B. Prade, and A. Mysyrowicz, Phys. Rev. Lett. 86, 5470 (2001).

${ }^{20}$ Q. Feng et al., IEEE J. Quantum Electron. 33, 127 (1997).

${ }^{21}$ S. L. Chin, A. Brodeur, S. Petit, O. G. Kosareva, and V. P. Kandidov, J. Nonlinear Opt. Phys. Mater. 8, 121 (1999).

${ }^{22}$ W. Liu, S. Petit, A. Becker, N. Akozbek, C. M. Bowden, and S. L. Chin, Opt. Commun. 202, 189 (2002).

${ }^{23}$ J. R. Fienup, J. Opt. Soc. Am. A 4, 118 (1987).

${ }^{24}$ A. Couairon, S. Tzortzakis, L. Berge, M. Franco, B. Prade, and A. Mysyrowicz, J. Opt. Soc. Am. B 19, 1117 (2002).

${ }^{25}$ M. Mlejnek, M. Kolesik, J. V. Moloney, and E. M. Wright, Phys. Rev. Lett. 83, 2938 (1999).

${ }^{26}$ W. Liu, O. Kosareva, I. S. Golubtsov, A. Iwasaki, A. Becker, V. P. Kandidov, and S. L. Chin, Appl. Phys. B: Lasers Opt. 76, 215 (2003).

${ }^{27}$ R. A. Ganeev, A. I. Ryasnyansky, M. Baba, M. Suzuki, N. Ishizawa, M. Turu, S. Sakakibara, and H. Kuroda, Appl. Phys. B: Lasers Opt. 78, 433 (2004). 